\title{
Recurrent Pulmonary Tuberculosis in Low Prevalence Settings: Case Report from Louisiana
}

\section{Amit Barua, Joseph Hubble, Kathryn Yoo, Michael Lacassagne, John P. Areno, Louis Trachtman and Juzar Ali*}

Department of Health, Office of Public Health, Tuberculosis Control, Louisiana, USA

*Corresponding Author: Juzar Ali, LSUHSC, \& Office of Public Health, Tuberculosis Control, Louisiana, USA.
Received: June 09, 2021

Published: August 16, 2021

(C) All rights are reserved by Michael

Lacassagne and Juzar Ali., et al.

\begin{abstract}
Recurrence of Pulmonary tuberculosis (TB) after successful completion of supervised treatment may be due to relapse or reinfection. Recurrence is common with an incidence rate of 2.26 per 100 person years over a mean follow up of 2.3 years. However, it is uncommon for an immunocompetent individual to experience multiple episodes of recurrences. We describe a 67yo, Human Immunodeficiency Virus (HIV) seronegative man, seen in the public health system in Louisiana, USA with multiple relapses of TB due to drug susceptible MTB despite successful completion of guideline based direct observed treatment regimen in each episode. This case report highlights the need for increased awareness and recognition of risk factors contributing to the recurrence of TB even in countries with low prevalence of TB. Better understanding of the importance of drug penetration and action on varying mycobacterial populations within diseased lung tissue would also aid clinicians in the management of these relapses.
\end{abstract}

Keywords: Recurrent Tuberculosis; Pulmonary Tuberculosis; Relapse; Low TB Prevalence Area

\section{Background}

Recurrent tuberculosis (TB), defined as the recurrence of TB after the successful completion of treatment, occurs due to 2 possible mechanisms: the subsequent infection caused by the same strain of Mycobacterium tuberculosis (MTB) commonly referred to as relapse, or that caused by a different genotype strain referred to as reinfection. However, relapse and reinfection cannot be distinguished clinically, hence, molecular genotyping methods such as Restriction Fragment Length Polymorphism (RFLP), spoligotyping, mycobacterial interspersed repetitive units (MIRU) typing or Whole Genome Sequencing (WGS) are employed to distinguish reinfection from relapse. Distinction between the two may be supported when genotyping is taken into consideration with epidemiological evidence [1]. Recurrence is common with an incidence rate of 2.26 per 100 person years over a mean follow up of 2.3 years [2]. However, it is uncommon for an individual to experience multiple episodes of recurrences in a lifetime. We describe a 67yo, Human Immunodeficiency Virus (HIV) seronegative man with multiple relapses of TB due to drug susceptible MTB seen in the public health system clinic in Louisiana, USA.

\section{Case Report}

A 67yo US-born, Caucasian male, previous smoker, ex-boiler maker and sandblaster with the past medical history of severe Chronic Obstructive Pulmonary Disease (COPD) and bronchiectasis presented with weight loss, productive cough and fever in 2012. He had an exposure history to 3 family members with active MTB disease. He was diagnosed with culture positive pulmonary disease and started on guideline-based standard directly observed regimen of Rifampin, Isoniazid, Pyrazinamide and Ethambutol. His treatment was completed in 6 months following sputum culture conversion and clinical improvement.

Subsequently he presented again in 2018 with respiratory symptoms and positive sputum culture with drug sensitive MTB for which he was retreated with extended treatment. He then had documented relapses in 2019 and 2021. This was despite adequate directly observed therapy with standard anti-TB drugs for extended 
duration and sputum conversion during the course and duration of these episodes. No additional exposure to MTB was identified throughout this period. Throughout these episodes of relapse, the patient was also managed for his COPD with triple drug inhaled therapy including long-acting beta-agonist, long-acting muscarinic antagonists and inhaled corticosteroids. He also required oral and injectable steroid boosts for episodes of acute exacerbation of COPD.

The isolates of all his relapses in 2012, 2018 and 2021 matched on Genotype analysis with Spoligotyping. The isolate submitted in 2019 was not tested due to contamination. In 2020 whole genome sequencing (WGS) was initiated as the new standard for genotyping. WGS compares $99 \%$ of the genotype compared to the 24 points of identification with spoligotyping. WGS analysis was performed on the 2018 and 2021 isolates, and the results matched. The combination of spoligotype and WGS results, in combination with the epidemiologic evidence, strongly supports that this patient has had disease relapse not disease reinfection.

Serum drug concentration were periodically monitored ensuring optimum dosage through these periods of retreatment. The radiological images throughout these episodes showed marginal improvement in nodular and cavitary infiltrates but without significant change in the bronchiectasis picture.

\section{Discussion}

The majority of the recurrences of MTB worldwide are caused by relapses, which occur in the first year after completion of treatment compared to reinfection which occur years later [2]. In the US, TB cases attributed to recurrence was $4-6 \%$ per annum [3]. The rate of recurrence depends upon the possibility of exposure to different strains of the organism and the susceptibility of the host for developing the disease [4]. The risk of recurrence is directly proportional to the background incidence of tuberculosis with low prevalence countries reporting lower rate of recurrence compared to those with high prevalence.

In countries with low prevalence, relapse is the primary cause of recurrence, whereas in countries with high prevalence, recurrence is mainly caused by reinfection [2]. Risk factors associated with recurrence include host factors such as male sex, cigarette smoking, low socioeconomic status, immigrants; underlying comorbidities such as HIV, Diabetes Mellitus (DM), chronic lung disease; and clinical factors such as low body weight, pretreatment positive sputum smear, pretreatment lung cavitation; and poor adherence to treatment regimen [5].

In the case of this patient, history of smoking could be one possible explanation for the multiple recurrence. Besides being at high risk of developing TB, smokers are at higher risk of developing recurrence compared to non-smokers [5]. Smoking prevents the macrophages from migrating to the site of infection and eliminating the organism resulting in poor treatment outcome and increased risk of delayed sputum smear or culture conversion [6].

With regards to environmental factors and occupational exposure, this patient is at increased risk of developing silico-tuberculosis having worked in the sandblasting industry for many years. Risk of developing TB is increased several fold in silicosis possibly due to the defective macrophages or the entrapment of MTB in the silicosis nodules [7].

True relapse occurs due to persistence of tuberculous bacilli [8]. This could be due to number of factors such as incorrect or ineffective dosing regimen, non-adherence to treatment, resistance to one or more anti-TB drugs or poor quality of drugs [9]. This patient was initiated on American Thoracic Society/Centers for Disease Control and Prevention/Infectious Diseases Society of America (ATS/ CDC/IDSA) directed TB treatment guidelines which dictate the prolongation of the 4-drug regimen with Directly Observed Therapy Strategy (DOTS) in recurrent TB caused by drug sensitive MTB isolates [10]. Standard Drug Sensitivity Tests (DST) performed in each episode of tuberculosis ruled out drug resistance, hence, the drug regimen was kept unchanged, although the duration of treatment was extended. Nevertheless, optimum therapeutic efficacy of drugs may not be reached due to variety of host factors including poor drug penetration in the lung tissue despite adequate serum drug levels [11]. Cavitary pulmonary disease is associated with high bacterial load resulting in $20 \%$ increase in the risk of relapse [10].

It is postulated that MTB may exhibit tolerance and persistence with metabolic slowdown and bacterial dormancy in adverse environmental conditions such as prolonged antibiotic exposure. This dormant population of bacteria are susceptible to antibiotics on 
standard DST, but are not cleared by antibiotics [12]. Mitchison hypothesized regarding four different populations of MTB occurring concurrently in a tuberculous lesion, varying in growth rate and metabolic activity. Despite the multiple drug regimen working on different population of bacteria, no antibiotic is completely effective against the completely inactive/dormant population [13]. This population of bacteria is considered to play an important role in recurrent infections [12].

Despite adequate drug levels in serum, it can be postulated that penetration of anti-TB drugs may have been less than optimum in this patient with COPD, bronchiectasis and cavitary disease. Higher doses of anti-TB drugs are associated with increased chance of culture conversion [14]. Injectable antibiotic such as amikacin is effective in eliminating MTB regardless of the metabolic state [15]. We postulate that these relapses are not due to multi-drug resistance, instead, they represent the ineffectiveness of the multi-drug regimen which may justify the use of additional drugs in the treatment portfolio if high risk recurrences occur. Hence, we believe higher doses of TB medications especially isoniazid and rifampin should be used for an extended duration. Additionally, a supplemental injectable antibiotic may be needed in such cases for optimum and sustained drug penetration into cavities and possible mycobacterial elimination.

\section{Conclusion}

Recurrent TB may not be unusual in countries with high prevalence of TB. However, this case report highlights the need for increased awareness and recognition of factors which may contribute to identifying recurrence of TB in low prevalent countries. Further research about drug penetration and action on varying mycobacterial populations with diseased lung tissue will also improve our understanding of treatment of MTB to prevent recurrences and achieve sustained cure rates.

\section{Funding Sources}

No funding was received in the publication of this article.

\section{Conflicts of Interest}

The authors declare that they have no conflicts of interest.

\section{Ethics Approval and Consent to Participate}

Not required/IRB exempt.
Bibliography

1. Genotyping | Data and Statistics | TB | CDC (2021).

2. Vega V., et al. "Recurrent TB: a systematic review and metaanalysis of the incidence rates and the proportions of relapses and reinfections". Thorax 76.5 (2021): 494-502.

3. Kim L., et al. "Epidemiology of recurrent tuberculosis in the United States, 1993-2010". International Journal of Tuberculosis and Lung Disease 17.3 (2013): 357-360.

4. Jasmer RM., et al. "Recurrent Tuberculosis in the United States and Canada". American Journal of Respiratory and Critical Care Medicine 170.12 (2004): 1360-1366.

5. Rosser A., et al. "Recurrent tuberculosis in the pre-elimination era". International Journal of Tuberculosis and Lung Disease 22.2 (2018): 139-150.

6. Wang EY., et al. "The impact of smoking on tuberculosis treatment outcomes: a meta-analysis". International Journal of Tuberculosis and Lung Disease 24.2 (2020): 170-175.

7. Farazi A and Jabbariasl M. "Silico-tuberculosis and associated risk factors in central province of Iran". Pan African Medical Journal 20 (2015).

8. Lambert M-L., et al. "Recurrence in tuberculosis: relapse or reinfection?" Lancet Infectious Disease 3.5 (2003): 282-287.

9. Cox H., et al. "Tuberculosis Recurrence and Mortality after Successful Treatment: Impact of Drug Resistance". PLoS Medicine 3.10 (2006): 384 .

10. Nahid P., et al. "Official American Thoracic Society/Centers for Disease Control and Prevention/Infectious Diseases Society of America Clinical Practice Guidelines: Treatment of Drug-Susceptible Tuberculosis". Clinical Infectious Disease 63.7 (2016): e147-e195.

11. Xu Y., et al. "Treating tuberculosis with high doses of anti-TB drugs: mechanisms and outcomes". Annals of Clinical Microbiology and Antimicrobials 16 (2017).

12. Meylan S., et al. "Targeting Antibiotic Tolerance, Pathogen by Pathogen”. Cell 172.6 (2018): 1228-1238.

13. Mitchison DA. "Basic Mechanisms of Chemotherapy". Chest 76.6 (1979): 771-780. 
14. Steingart KR., et al. "Higher-dose rifampin for the treatment of pulmonary tuberculosis: a systematic review". International Journal of Tuberculosis and Lung Disease 15.3 (2011): 305-316.

15. de Steenwinkel JEM., et al. "Time-kill kinetics of anti-tuberculosis drugs, and emergence of resistance, in relation to metabolic activity of Mycobacterium tuberculosis". Journal of Antimicrobial Chemotherapy 65.12 (2010): 2582-2589.

Volume 5 Issue 9 September 2021

(C) All rights are reserved by Amit Barua., et al. 